\title{
INDEX TO VOLUME 67
}

\section{RESEARCH ANNOUNCEMENTS}

Auslander, Louis. A characterization of discrete solvable matrix groups, 235.

On the Euler characteristic of compact complete locally affine spaces. II, 405.

Auslander, L., Green, L. and Hahn, F. Flows on some three dimensional homogeneous spaces, 494.

Auslander, L., Hahn, F. and Markus, L. Topological dynamics on nilmanifolds, 298.

Barry, P. D. The minimum modulus of integral functions of small order, 231.

Bellman, Richard and Karush, William. On a new functional transform in analysis: The maximum transform, 501.

Bellman, Richard, Kalaba, Robert and Wing, G. M. Invariant imbedding and variational principles in transport theory, 396.

Bers, Lipman. Holomorphic differentials as functions of moduli, 206.

- Correction to "Spaces of Riemann surfaces as bounded domains," 465.

Bishop, Errett and Phelps, R. R. A proof that every Banach space is sub-reflexive, 97.

Block, H. D. and Fuchs, W. H. J. An enclosure theorem for eigenvalues, 425.

Borel, Armand. Some properties of adele groups attached to algebraic groups, 583.

Borel, Armand and Harish-Chandra. Arithmetic subgroups of algebraic groups, 579.

de Branges, Louis. Some Hilbert spaces of entire functions, 129.

Browder, Andrew. Cohomology of maximal ideal spaces, 515.

Browder, F. E. Approximation in uniform norm by solutions of elliptic differential equations, 400.

Cairns, S. S. The manifold smoothing problem, 237.

- A simple triangulation method for smooth manifolds, 389.

Carleson, Lennart. A remark on Picard's theorem, 142.

Cartier, P. On H. Weyl's character formula, 228.

Chacon, R. V. On the ergodic theorem without assumption of positivity, 186.

Chandrasekharan, K. and Narasimhan, Raghavan. On Hecke's functional equation, 182.

Cohen, Eckford. Fourier expansions of arithmetical functions, 145.

Connell, E. H. See Porcelli, P.

Curtis, P. C., Jr. Derivations of commutative Banach algebras, 271.

Dade, E. C., Taussky, O. and Zassenhaus, H. On the semigroup of ideal classes in an order of an algebraic number field, 305.

Dean, R. A. Coverings in free lattices, 548.

Dombrowski, Peter. On global solutions for partial differential equations of first order, 202.

Edwards, C. H., Jr. Concentric tori in the 3-sphere, 220.

Ehrenpreis, Leon. A new proof and an extension of Hartog's theorem, 507.

Ernest, John. A decomposition theory for unitary representations of locally compact groups, 385.

Fadell, Edward and Van Buskirk, James. On the braid groups of $E^{2}$ and $S^{2}, 211$.

Faith, Carl. Derivations and generations of finite extensions, 550.

Fary, I. Dimension of the square of a space, 135.

Fuchs, W. H. J. See Block, H. D.

Gluck, Herman. The embedding of two-spheres in the four-sphere, 586.

Orientable surfaces in four-space, 590.

Goffman, Casper. Area of discontinuous surfaces, 309. 
Grant, K. and Whaples, G. Abstract class formations, 393.

Green, L. W. Proof of Blaschke's sphere conjecture, 156.

Spectra of nilflows, 414.

See Auslander, L.

Haefliger, André. Differentiable imbeddings, 109.

Hahn, F. See Auslander, L.

Harish-Chandra. See Borel, Armand.

Hausner, Alvin. On a homomorphism between generalized group algebras, 138.

Hayman, W. K. and Wilansky, Albert. An example in summability, 554.

Heins, Maurice. A class of conformal metrics, 475.

Helms, L. L. A representation of the infinitesimal generator of a diffusion process, 479.

Hsiang, F. C. Summability $(L)$ of Fourier series, 150.

Huebsch, William and Morse, Marston. Conical singular points of diffeomorphisms, 490.

Ionescu Tulcea, C. Spectral operators on locally convex spaces, 125.

Jerrard, Richard. Curvature and length, 113.

Jung, H. A. Kennzeichnung Г-primer Graphen, 463.

Kalaba, Robert. See Bellman, Richard.

Karlin, Samuel. Total positivity, absorption probabilities and applications, 105.

Karush, William. See Bellman, Richard.

Kemeny, J. G. and Snell, J. L. Potentials for denumerable Markov chains, 120.

Kister, J. M. Examples of periodic maps on Euclidean spaces without fixed points, 471.

Klee, Victor. Topological equivalence of a Banach space with its unit cell, 286.

Klingenberg, Wilhelm. Orthogonal groups over local rings, 291.

Kobayashi, Shoshichi. Compact Kaehler manifolds with positive Ricci tensor, 412.

Koppelman, Walter. Boundary value problems for pseudoanalytic functions, 371.

Transmission problems in function theory, 565.

Krabbe, G. L. Integration with respect to operator-valued functions, 214.

Kristensen, Leif. On the cohomology of two-stage Postnikov systems, 597.

Kuiper, N. H. A continuous function with two critical points, 281.

Kunze, R. A. and Stein, E. M. Analytic continuation of the principal series, 593.

Laha, R. G. On a property of functions with non-negative derivatives at the origin and its application, 148.

Lamperti, John. A new class of probability limit theorems, 267.

Lazerson, E. E. Onto inner derivations in division rings, 356.

Lehner, Joseph. Magnitude of the Fourier coefficients of automorphic forms of negative dimension, 603.

McCarthy, G. S., Jr. Local connectivity in homeomorphism groups, 420.

McMillan, D. R., Jr. Cartesian products of contractible open manifolds, 510.

Machover, M. The theory of transfinite recursion, 575.

Marcus, Marvin and Newman, Morris. The permanent function as an inner product, 223.

Markus, L. See Auslander, L.

Massey, W. S. Obstructions to the existence of almost complex structures, 559.

Mazur, Barry. Stable equivalence of differentiable manifolds, 377.

Mejlbo, L. C. and Schmidt, P. F. On the determinants of certain Toeplitz matrices, 159.

Morse, Marston. See Huebsch, William.

Mumford, David. An elementary theorem in geometric invariant theory, 483.

Narasimhan, Raghavan. See Chandrasekharan, K.

Newman, Morris. See Marcus, Marvin.

Nunke, R. J. Slender groups, 274. 
Oglesby, F. C. Report: An examination of a decision procedure, 300.

Orey, Steven. Strong ratio limit property, 571.

Palais, R. S. Equivalence of nearby differentiable actions of a compact group, 362.

Parter, S. V. On the extreme eigenvalues of truncated Toeplitz matrices, 191.

Peck, L. G. Simultaneous rational approximations to algebraic numbers, 197.

Phelps, R. R. See Bishop, Errett.

Porcelli, P. and Connell, E. H. A proof of the power series expansion without Cauchy's formula, 177.

Quine, W. V. A basis for number theory in finite classes, 391.

Ranga Rao, R. On the central limit theorem in $R_{k}, 359$.

Redheffer, Raymond. A new maximum principle, 219.

Ree, Rimhak. A family of simple groups associated with the simple Lie algebra of type $\left(F_{4}\right), 115$.

Reiner, Irving. The Krull-Schmidt theorem for integral group representations, 365.

Rieger, G. J. On the prime ideals of smallest norm in an ideal class mod $f$ of an algebraic number field, 314.

Rota, Gian-Carlo. On the eigenvalues of positive operators, 556.

Sacks, G. E. A minimal degree less than 0', 416.

Schaefer, H. H. A new class of spectral operators, 154.

Schaerf, H. M. On the existence of invariant measures, 504.

Schmidt, P. F. See Mejlbo, L. C.

Słowikowski, W. On continuity of inverse operators, 467. Quotient spaces and the open map theorem, 498.

Smith, K. T. Inequalities for formally positive integro-differential forms, 368 .

Snell, J. L. See Kemeny, J. G.

Stanek, P. F. G. Two element generation of the symplectic group, 225.

Stein, E. M. On some functions of Littlewood-Paley and Zygmund, 99.

The characterization of functions arising as potentials, 102.

See Kunze, R. A.

Steinberg, Robert. A general Clebsch-Gordan theorem, 406.

$\mathrm{Su}, \mathrm{J}$. C. On a problem of P. A. Smith, 422.

Swanson, C. A. An inequality for linear transformations with eigenvalues, 607

Taussky, O. See Dade, E. C.

Toda, Hirosi. Vector fields on spheres, 408.

Van Buskirk, James. See Fadell, Edward.

Varadarajan, V. S. Convergence of stochastic processes, 276.

Wallace, A. D. A theorem on acyclicity, 123.

Whaples, G. See Grant, K.

Wilansky, Albert. See Hayman, W. K.

Wilf, $\mathrm{H}$. S. The argument of an entire function, 488.

Wing, G. M. See Bellman, Richard.

Zassenhaus, H. See Dade, E. C.

Zeeman, E. C. Knotting manifolds, 117.

The generalised Poincare conjecture, 270.

\section{RESEARCH PROBLEMS}

Aumann, R. J. Extending an order, 176.

Bellman, Richard. Generalized exponentials and Baker-Hausdorff-Campbell series, 176. Asymptotic control theory, 262.

Differential equations, 262. 
Control processes, 456.

Theory of numbers, 456.

Commutative Banach algebra problems. A list of problems assembled in a conference on commutative Banach algebras held at Dartmouth College in August, 1960, 457.

Harary, Frank. 542.

Mullin, A. A. Properties of mutants, 82.

Wilansky, Albert. An elementary inequality, 355.

Wilf, H. S. Reciprocal bases for the integers, 456.

\section{REPORTS OF MEETINGS AND MISCELLANEOUS ARTICLES}

Council and Board of Trustees-1960, 163.

Doctorates conferred in 1960, 316.

Green, J. W. See Pitcher, Everett.

See Paige, L. J.

Huff, G. B. Reports of Meetings of the American Mathematical Society: The November meeting in Nashville, 173.

Meder, A. E., Jr. Report of the Treasurer, 264.

Paige, L. See Pitcher, Everett.

Paige, L. J., Green, J. W. and Pitcher, Everett. Reports of Meetings of the American Mathematical Society: The Annual Meeting in Washington, 251.

Paige, L. J. and Youngs, J. W. T. Reports of Meetings of the American Mathematical Society: The Summer Meeting in Stillwater, 543.

Pierce, R. S. Reports of Meetings of the American Mathematical Society: The November Meeting in Pasadena, 174; The April Meeting in Stanford, 354; The June Meeting in Seattle, 461.

Pitcher, Everett. Reports of Meetings of the American Mathematical Society: The October Meeting in Worcester, 81; The February Meeting in New York, 261. See Paige, L. J.

Pitcher, Everett and Paige, L. J. Reports of Meetings of the American Mathematical Society: The April Meeting in New York, 346.

Youngs, J. W. T. Reports of Meetings of the American Mathematical Society: The November Meeting in Evanston, 175; The April Meeting in Chicago, 353.

- See Paige, L. J.

\section{BOOK REVIEWS}

Aharoni, J. The special theory of relativity. Alfred Schild, 83.

Ahlfors, L. V. See Nevanlinna, R.

Ahlfors, L. V. and Sario, Leo. Riemann surfaces. George Springer, 170.

Barrett, J. H. See Petrovskii, I. G.

Behnke, H. See Nevanlinna, R.

Bergman, Stefan. See Bers, Lipman.

Bers, Lipman. Mathematical aspects of subsonic and transonic gas dynamics. Stefan Bergman, 337.

See Nevanlinna, R.

Billingsley, Patrick. See Meyer-Eppler, W.

Borel, A. Seminar on transformation groups. P. E. Conner, 450.

Borsuk, K. and Szmielew, Wanda. Foundations of geometry, Euclidean and BolyaiLobachevskian geometry, projective geometry. Hans Freudenthal, 342.

Botts, T. A. See McShane, E. J.

Brenner, J. L. See Gantmacher, F. R.

Bruck, R. H. A survey of binary systems. Erwin Kleinfeld, 340. 
Cartwright, M. L. Integral functions. J. Korevaar, 454.

Cassels, J. W. S. An introduction to the geometry of numbers. L. J. Mordell, 89.

Conner, P. See Borel, A.

Császár, Ákos. Fondements de la topologie générale. Leonard Gillman, 538.

Dieudonné, J. Foundations of modern analysis. Leopoldo Nachbin, 246.

Doob, J. L. See Loève, Michel.

Erdélyi, A. See Tricomi, F. G.

Fan, Ky. See Schatten, Robert.

Freudenthal, Hans. See Borsuk, K.

- See Segre, B.

Gantmacher, F. R. The theory of matrices, trans. by K. A. Hirsch. Jack McLaughlin; Applications to the theory of matrices, trans. by J. L. Brenner. Jack McLaughlin, 95.

Gillman, Leonard. See Császár, Ákos.

Goldberg, R. R. Fourier transforms. Walter Rudin, 440.

Gottschalk, W. H. See Nemytskii, V. V.

Grauert, H. See Nevanlinna, R.

Grothendieck, A. Eléments de géométrie algébrique. S. Lang, 239.

Hannan, E. J. Time series analysis. M. Rosenblatt, 535.

Heins, M. See Nevanlinna, R.

Hirsch, K. A. See Gantmacher, F. R.

James, R. C. See Taylor, A. E.

Jenkins, J. A. See Nevanlinna, R.

John, Fritz. See Sauer, Robert.

Kamel, H. See Petrovskii, I. G.

Kleinfeld, Erwin. See Bruck, R. H.

Kodaira, K. See Nevanlinna, R.

Komm, H. See Petrovskii, I. G.

Korevaar, J. See Cartwright, M. L.

Lang, S. See Grothendieck, A.

Levinson, Norman. See Nemytskii, V. V.

Loève, Michel. Probability theory. J. L. Doob, 446.

Lombardo-Radice, L. See Segre, B.

McLaughlin, Jack. See Gantmacher, F. R.

McShane, E. J. and Botts, T. A. Real analysis. J. M. H. Olmsted, 86.

Meyer-Eppler, W. Grundlagen und Anwendungen der Informationstheorie. Patrick Billingsley, 89.

Mordell, L. J. See Cassels, J. W. S.

Nachbin, Leopoldo. See Dieudonné, J.

Nemytskii, V. V. and Stepanov, V. V. Qualitative theory of differential equations. W. H. Gottschalk and Norman Levinson, 443.

Nevanlinna, R., Behnke, H., Grauert, H., Ahlfors, L. V., Spencer, D. C., Bers, L., Kodaira, K., Heins, M. and Jenkins, J. A. Analytic functions. Hugo Rossi, 533.

Niven, Ivan and Zuckerman, H. S. An introduction to the theory of numbers. A. L. Whiteman, 339.

Northcott, D. G. An introduction to homological algebra. Alex Rosenberg, 440.

Olmsted, J. M. H. See McShane, E. J.

O'Meara, O. T. See Watson, G. L.

Ostrowski, A. Vorlesungen über Differential- und Integralrechnung. Vol. I, Funktionen einer Variablen. 2nd. rev. ed. A. E. Taylor, 336.

Petrovskii, I. G. Lectures on the theory of integral equations, trans. by H. Kamel and H. Komm. J. H. Barrett, 333. 
Rickart, C. E. General theory of Banach algebras. Bertram Yood, 334.

Rindler, W. Special relativity. Alfred Schild, 449.

Rosenberg, Alex. See Northcott, D. G.

Rosenblatt, M. See Hannan, E. J.

Rossi, Hugo. See Nevanlinna, R.

Rudin, Walter. See Goldberg, R. R.

Sario, Leo. See Ahlfors, L. V.

Sauer, Robert. Anfangswertprobleme bei partiellen Differentialgleichungen. Fritz John, 171.

Schatten, Robert. Norm ideals of completely continuous operators. Ky Fan, 532.

Schild, Alfred. See Aharoni, J.

See Rindler, W.

Segre, B. Lectures on modern geometry. Appendix by L. Lombardo-Radice. Hans Freudenthal, 442.

Sikorski, Roman. Funkcje Rzeczywiste (Real functions), vol. II. Stanislaw Ulam, 172.

Spencer, D. C. See Nevanlinna, R.

Springer, George. See Ahlfors, L. V.

Stepanov, V. V. See Nemytskii, V. V.

Szmielew, Wanda. See Borsuk, K.

Taylor, A. E. Introduction to functional analysis. R. C. James, 344.

See Ostrowski, A.

Tricomi, F. G. Vorlesungen über Orthogonalreihen. A. Erdélyi, 447.

van der Waerden, B. L. Algebra. Part II. Daniel Zelinsky, 83.

Watson, G. L. Integral quadratic forms. O. T. O'Meara, 536.

Whiteman, A. L. See Niven, Ivan.

Yood, Bertram. See Rickart, C. E.

Zelinsky, Daniel. See van der Waerden, B. L.

Zuckerman, H. S. See Niven, Ivan.

\section{INVITED ADDRESSES}

Abhyankar, Shreeram, 81.

Apostol, T. M., 462

Dolph, C. L. Recent developments in some non-self-adjoint problems of mathematical physics, 1.

Fuller, F. B. Fixed points of multiple-valued transformations, 165.

Halmos, P. R. Recent progress in ergodic theory, 70.

Herstein, I. N., 353.

- Lie and Jordan structures in simple, associative rings, 517.

Higman, Graham, 175.

Hörmander, Lars, 251.

Jenkins, J. A., 353.

Kan, D. M., 346.

Royden, Halsey, 174.

Stoker, J. J., 251.

Wermer, John, 261.

Wielandt, Helmut, 251.

Wolfowitz, Jacob, 346.

Young, G. S., Jr., 173.

Zassenhaus, Hans, 354.

- Modern developments in the geometry of numbers, 427. 